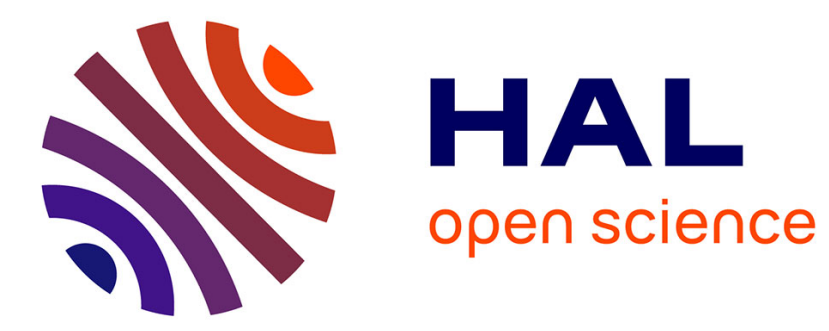

\title{
Towards computer-aided text production
}

\author{
A. Bisseret
}

\section{To cite this version:}

A. Bisseret. Towards computer-aided text production. RR-0665, INRIA. 1987. inria-00075888

\section{HAL Id: inria-00075888 \\ https://hal.inria.fr/inria-00075888}

Submitted on 24 May 2006

HAL is a multi-disciplinary open access archive for the deposit and dissemination of scientific research documents, whether they are published or not. The documents may come from teaching and research institutions in France or abroad, or from public or private research centers.
L'archive ouverte pluridisciplinaire HAL, est destinée au dépôt et à la diffusion de documents scientifiques de niveau recherche, publiés ou non, émanant des établissements d'enseignement et de recherche français ou étrangers, des laboratoires publics ou privés. 


\section{Rapports de Recherche}

UNITÉ DE RECHERCHE INRIA-ROCOUENCOURT
$N^{\circ} 665$

\section{TOWARDS COMPUTER - AIDED TEXT PRODUCTION}

Institut National de Recherche en Informatique et en Automatique

Domaine de Voluceau Rocquencourt BP 105

78153 Le Chesnay Cedex France Tél.:(1) 39635511 
TOWARDS COMPUTER-AIDED TEXT PRODUCTION

\title{
VERS LA PRODUCTION DE TEXTE ASSISTEE PAR ORDINATEUR
}

\author{
André BISSERET
}

Avril 1987

This paper is a revised version of the keynote address to the Third European Conference on Cognitive Ergonomics, Paris, 15-19 September 1986. 


\section{Résumé}

A partir d'une revue de résultats de recherches expérimentales sur les modèles de planification et sur différentes activités de conception chez l'homme, cet article esquisse ce que pourrait être une aide informatique à la production de texte. Plutôt que sur l'édition l'accent est mis sur le sous-processus de planification de l'activité de composition ainsi que sur le sous-processus de mise en phrases.

On insiste sur la nécessaire distinction entre le plan du produit qui est hiérarchique et le plan de l'activité de production qui, elle, est "opportuniste". On montre que les éditeurs de plan actuels n'assistent pas cette activité parce, du fait qu'ils sont fondés sur un modèle hiérarchique, ils imposent un processus descendant. Ils rendent très difficiles les processus ascendants inhérents à l'activité de conception. Des exemples de systèmes expérimentaux qui assisteraient réellement cette activité sont donnés.

Mots clés: Sciences Cognitives; Processus de conception; Processus de Planification; Production de Texte; Assistance Informatique.

\section{Summary}

This paper reviews the main results of experimental researches on planning activity models and on different human design activities in order to sketch possible ways to assist by computer the whole writing process. Rather than on editing, the focus is mainly on the planning sub-process and also on translating.

The paper emphazises the distinction between the plan of the product which is hierarchical and the plan of the activity which is "opportunist". It is assumed that the current plan editors do not really assist the activity because, as they are founded on a hierarchical model, they require a top-down process. They hinder the bottom-up aspects of the composing activity. Exemples of experimental systems which would better support this activity are given.

Key-words: Cognitive Sciences; Design Process; Planning Process; Writing Process; Computer Aid. 
TOWARDS COMPUTER-AIDED TEXT PRODUCTION

\section{INTRODUCTION}

One of the characteristic phenomena of the design process, and it is one which appears particularly during the first phase of the process, is the changing of objectives.

The process of preparing this paper proved to be no exception: my first objective, decided about six months before this version, was reflected by the title given at that time: "The Psychology of Production". But when I started to prepare this text, I modified this too general objective and was thus forced to change the title!

Thus this text remains about the production process and possible ways to assist it, but it is mainly focused on the specific field of text production.

Writing is, of course, an extremely widespread task, and yet at the same time, it is probably one of the least aided tasks.

Being researchers, we are among the first to be interested by the possibility of computer-aided writing systems. In fact, as "generating ideas" is part of the total writing process, we are almost always in the process of creating texts.

But industry, towards which we direct our ergonomic work, is also a great producer of texts. Machine manufacturers, and in particular computer constructors, are also great producers of printed paper - the number of pages of manuals that they publish each year is enormous.

Computer-aided systems have already been introduced in other areas of design: CAO systems in technical design departments and architects'offices, and programming environments for computer programmers. But how far can we go towards the design of a work station for writers, and how can cognitive ergonomics contribute to its specification?

This is the problem that I shall be discussing here, bringing together a certain number of results drawn not only from research into Psychology and Psycholinguistics, but also from informatics of aiding software and Artificial Intelligence.

\section{Production and reception}

My preliminary remark is that, among research into cognitive activities, production is studied to a much lesser degree than reception, and this is particularly noticeable in the field of texts. Whereas there has been a great deal of research into text comprehension, research into text production has been much less substantial and this is true as much in Psychology as in Artificial Intelligence. 
In the case of Psychology, Gould (1980) explains this as a consequence of a greater methodological difficulty: experimental paradigms based on comprehension tasks can be applied more easily than those based on production tasks.

Perhaps it has also been too readily assumed, at a theoretical level, that if the comprehension activity could be explained, then an explanation of the production activity will follow automatically from this.

In fact production is quite different from comprehension, even if they have some cognitive processes in common, and. more research should be undertaken into the production process.

\section{The top-down assumption}

The production process has been, and is still considered as being based on a top-down model. This belief is shared by the layman and by specialists in the organization of work. Very often it is represented as a sequence of phases where the output of one phase becomes the input for the next. Here is one example, which was chosen almost at random from a good number of others:

- preliminary study

- detailed study

- technical study

- implementation

- test

- utilization

- maintenance

More generally, in this perspective, designing and producing something mean defining an objective as a starting point, and breaking this objective down into a series of sub-objectives. These, in turn, are broken down into sub-subobjectives, and so on until a sequence of elementary actions is designed that will achieve the product.

In the field of text production, the same schema is often admitted. Nobody has forgotten the wise advice of teachers: "Start by making a plan, and everything will fall into place". But how can a plan be made?, and how can it be filled in?

As far as I am concerned, when preparing this text, I kept on finding that once the plan had been formed, it remained rather unstable, and moreover that writing different parts of the text is a stressful activity, full of shifts and movements, in ideas, on paper, on the screen and even.... up and down the corridor!

My plan for a first part is to start with some important results drawn from works on planning models. Then I will review briefily researches which have been carried out on different human design activities, in order to focus finally and in more detail, on the writing process itself and on the problem of its assistance by informatics. 


\section{THE RESEARCH ON PLANNING}

\section{Hierarchical Model}

Concerning planning models, it is true that an important line of research in Artificial Intelligence seems at a first sight to strengthen the common idea. This line has developed models of planning activity which are based on a completely top-down approach. These are the "Hierarchical Planners" the best-known example of which is NOAH (for Nets of Action Hierarchies) produced by Sacerdoti (1977).

This type of plan takes the form of a hierarchical tree.The root represents the general goal of the activity, and the leaves represent the sequence of concrete actions that enable the goal to be reached. Each level is a complete representation of the plan but with a decreasing degree of abstraction from the root to the leaves.

However, the process of constructing this sequence of actions is carried out, in a purely top-down way. The planner begins by breaking the general goal into a set of sub-goals which is the second level. These sub-goals are in turn refined in a third level etc. But each time a new level is created, a "criticism" is executed, in order to solve possible contradictions, interactions and/or redundancies. This technique, known as the "least commitment" technique, was introduced as a powerful means of minimizing 'U-turns', goal modifications and/or changes in the order of the goals.

It is understandable that this type of model satisfies the specialists in Artificial Intelligence as far as they do not attempt to simulate human processes nor to interact with a user in a planning task.

But as soon as we try to design tools for interactive aid, we come up against the problem of understanding, in order to take them into account, the differences between the logico-mathematically powerful representations used by program designers to maximise the power of their programs, and the various heuristic representations that people use in ordinary life, in order to adapt rapidly to different situations (see Bisseret, 1983).

\section{Opportunistic Plan}

In such a psychological perspective, Hayes Roth \& Hayes Roth (1979) carried out a very interesting analysis of verbal protocols from subjects who had the task of planning a set of errands, to be done in one day, in a town. The model they built from this was then implemented on a computer.

This model is more comprehensive than the strictly hierarchical model: it does not contradict it but it includes it as a special case of human planning. This is the case when the task inherently presents a strongly hierarchical structure and/or when the subjects are experts in the task. They may then use readymade plans that they already have at their disposal and that they need only to invoke and adapt to the particular situation.

In the other cases, Hayes Roth \& Hayes Roth clearly show the importance of the bottom-up process in the subjects' planning activity. They speak of 
opportunistic planning. Different levels of abstraction remain an important characteristic of this type of planning, but the subjects do not construct them according to a systematic refinement of the more abstract goal. The subjects work multi-directionally in the plane of abstraction. They frequently construct low-level sub-plans at the outset, which are relatively independent of each other and then combines them at a higher level of abstraction.

In particular the subjects can build sequences of elementary actions which are not in keeping with a more abstract plan. Thus they can work on a partial but seemingly promising aspect of the problem, without yet knowing how they will incorporate it into an overall solution.

The authors note that in doing so, the subjects run the risk of producing incoherencies that will have to be corrected afterwards: an eventuality which a purely top-down hierarchical planning avoids. But a specific advantage of the opportunistic strategy is that its bottom-up structure is an interesting source of innovation: low level decisions and connected observations can lead to the discovery of new high level plans.

\section{EXPERIMENTAL RESEARCH INTO DESIGN}

Now, I am going to show that the validity of the opportunistic model is supported by experimental researches on different human design activities.

For several years the IBM Behavioral Science Group has been studying design process in general in order to gain a greater understanding of the computer software design process (Malhotra et al., 1980; Thomas \& Carroll, 1981).

From this group, Carroll and Rosson (1985) have recently published a very interesting synthesis on the topic. They develop a convincing criticism of classical approaches, which they term "analytical approaches". They show that such approaches are more normative than experimental in that they represent the views of their authors "about what design should be like but fail to seriously consider what design activity is like in fact".

Carroll and Rosson underline two faults in these analytical approaches: the first one is that they consider design as being a top-down refinement of the problem in sub-problems, and secondly, for this approaches, "design appears to be a state instead of a process". They show in particular how recent, wellknown work on command languages, carried out by Moran (1981) and Reisner (1984), do not escape their criticism.

Carroll and Rosson; on the basis of precise empirical studies offer a radically different view of design activity which corroborates the results of Hayes Roth and Hayes Roth on opportunistic planning and which they summarize in the following way (Carroll and Rosson, op. cit. p. 27):

"- design is a process (it is not a state);

- design is non-hierarchical: neither strictly bottom-up nor strictly topdown; 
- design is radically transformational, involving the development of partial and interim solutions which may ultimately play no role in the final design;

- lastly design intrinsically involves the discovery of new goals"

Visser (1987) is studying the activity of a technician in the design department of a firm where machine-tools are made. This technician is provided with the specifications of a machine and he is supposed to translate these specifications into the form of "sequence-schemas" intended for the programmer of the automatic control system. The first protocol analyses show that the technician activity is far from being a simple translation of the specifications. Not only does he fill in gaps and correct errors but he also takes decisions that put in doubt previous decisions and may even lead to modifying the design of physical characteristics of the machine.

In a fine book on the cognitive psychology of planning, Hoc (1987) emphasizes the interaction between top-down and bottom-up processes. The author carried out a great deal of research into the cognitive activity of software designers. Particularly in this field the normative model of refining a problem into sub-problems tends to be put forward as being the solution. It is the panacea of "structured programming". Hoc's results clearly show that this method, however satisfying it might appear in itself, can hinder the subjects'normal strategies.

Thus, studies on design in different fields, when they rely on precise empirical data, all show that the real design process as carried out by a human subject does not correspond to the ideal image sought by many people. Specially designers of software for computer-aided tasks, generally, are too ready to believe that the activity of the future user is necessarily a "beautiful tree".

It is true that the product itself can generally be represented by a nice hierarchical plan, although it is sometimes arbitrary (Tazi \& Virbel, 1985b); but in any case, the plan of the product itself is one thing, the plan of the activity of production is quite another.

\section{THE HAYES \& FLOWER WRITING-PROCESS MODEL}

Let me now focus back on the text production process itself. I will first present the main characteristics of the writing model which has been proposed by Hayes \& Flower (1980) and then I will review in more detail different subprocesses and the possibilities of their computer assistance.

Hayes and Flower are the first authors to have studied the writing activity by mean of the method of "protocol analysis". Proceeding this way they had the opportunity to analyse, not only the product but mainly the process of its production.

The main characteristics of the model for our purpose are the following: the model identifies the process as composed of different sub-processes and shows the organization of these sub-processes. 
These are:

- the process of planning which is composed of the processes of idea generating, idea organizing, and goal setting;

- the process of translating ideas into the form of language

- and the editing process that involves correcting the text once it has been produced.

I would like to concentrate on the model's main dynamical characteristics that have been tested in a relatively precise way.

The authors show that these sub-processes are not organized as a strict sequence, from generating to editing. Actually in a first phase the more frequent sub-process is generating but it is interrupted from time to time by editing. In a second phase organizing is dominant but interrupted by generating and editing. In a third phase, translating dominates but is interrupted by generating and editing.

Moreover, these interruptions are both frequent and widely distributed.

In the model it is the role of a "monitor" to manage the interventions of the different sub-processes. The monitor makes it possible to simulate different writing strategies, corresponding to differences among individuals.

An important aspect, which should be emphasized, concerns the different types of physical traces that characterise each sub-process.

The generating process is characterized by the writing of notes which often consist of a single word or phrase and only seldom take the form of a complete sentence.

The organizing process is also characterized by the writing of notes but here they take on a more complex form: they include physical marks such as numbering or indenting, that organize at least two ideas. Two general structures are represented this way:

- either a temporal structure ("I'Il speak about design process in other domains and then about writing process")

- or/and a hierarchical structure (under "writing aid" l'll speak about translating and planning").

The goal setting process is characterized not by content, but by metacomments which evaluate the text. For example "it would be more interesting to give examples from my own writing activity" or "I'm needing a transition here".

The translating process is, of course, characterized by complete sentences that are intended to be included in the text. 
Finally, the editing process is characterized by all the corrections, both in the notes and in the text itself, and concern all levels of language from spelling mistakes to pragmatic and rhetorical changes.

In summary, I underline two main points:

Results on writing process are identical to those obtained in other areas of design. In fact writing is composed of several sub-processes which are not organised in a linear sequence of steps, but they interact throughout the writing activity.

However each of these sub-processes is clearly distinguishable. Each of them bring into play specific cognitive activities, which give rise to specific observable behavior.

Taking advantage of this, I shall now review in more detail two of these subprocesses: first the planning and second the translating process.

For each, calling upon studies I found relevant, I shall pose the problem of possible specific computer aids, which could be integrated in a future writer's work station.

\section{COMPUTER AIDED PLANNING}

As main components of the planning process the Hayes \& Flower model identify the two sub-processes of generating and organizing.

It should be said at the outset, that the term "generating" is really something of an over-exaggeration when it comes to the real process that is studied by research into writing. "Retrieval" is also used and I find it more appropriate.

Even having taken this precaution, describing at a fundamental level of this retrieval process is still a research problem. It is dependent on progress in several areas of cognitive psychology, such as research into memory, learning but also problem-solving.

That is a lot and I shall be satisfied here to simply underline several characteristics of the retrieval process, and more specifically its relation to the organizing process in so far as they are relevant to the problem of their computer assistance.

"From chaos to order" is the image that Brown and Newman (1985) used to sum up to the process of text production. But what is known of this chaos and the way it is ordered?"

Firstly, it is the sub-process that is the most time-consuming relative to the other components of the whole writing process. Gould (1980) in his study on letter writing, showed that planning accounted for two thirds, on average, of the total time spent. An efficient computer-aiding system would thus be really worthwhile.

A second point is the essential difference between the plan of the text and the plan of the activity. Hayes and Flower stress that the sequence of ideas retrieval is largely determined by a process of ideas association whereas the 
final text shows an obviously different plan which is plainly the result of the organizing process.

It is necessary thus to distinguish between the content plan and the process plan. The content plan is an abstraction of the complete text, whereas the process plan concerns the writer's strategies which is to say the goals that he sets and the means he uses to reach them.

This process plan gives rise to meta-comments that the writers make to themselves. The following is a significant example taken from one of the authors'protocols:

"I am really having a hard time getting started. Well, may be l'll just write a bunch of ideas down and may be try to connect them after".

Such a conscious control of the process itself appears to be a specific characteristic of good writers. On the contrary, novices too quickly try to produce and manipulate text instead of explicitly manipulating ideas (see also Collins \& Gentner, 1980).

Concerning possible ways to aid the retrieving and organizing process it is clear that computer expertise cannot be still seriously considered. However some kind of concrete support can be imagined which would allow the writers to materialize and manipulate their ideas on the screen. Such an hypothesis raises two questions.

It is clear that texts consist of more elementary, well defined units such as words, sentences and paragraphs that can be used as primitives for text editing systems. But what about idea as a unit? Is an idea an autonomous unit, a primitive that could be presented concretely in a computer-aided system for a direct manipulation of ideas?

And secondly what are the connections between ideas? Here again, is it possible and useful to provide the user with concrete presentations of them, and if so, to what level of detail?

\section{Representing ideas}

It seems realistic to consider ideas as units. On the one hand not only does the "idea of an 'isolable idea' "exist, but common observation, confirmed by studies on writers'protocols shows that actual behaviour corresponds with this. There are the one-word or single phrase notes that are often distributed over separate sheets of paper.

Moreover, research into text comprehension, particularly the model from Kintsch and Van Dijk (1978) contributes to precise the nature of ideas.

According to this model, the sentences of a text are translated by the readers into sequences of semantic propositions (that is to say predicate-argument units). These propositions constitute the "micro-structure" of the text.

Each proposition is interpreted not in an absolute way, but in relation to the others. As soon as the text reaches several dozen propositions, the readers 
develop a process of reducing the semantic information. They apply four rules, called "macro-rules". Each using a different procedure, these macro-rules allow the readers to eliminate the details of individual propositions and to construct chunks of signification, called "macro-propositions" which retain the global meaning of the text and which form a "macro-structure" (see Van Dijk, 1977).

This rules application process is recursive: as soon the first level of macrostructure contains too many macro-propositions, the readers (re)apply the rules to obtain macro-structures at a second level of generality, and so on.

This is the process that allows us remember for example, the content of a five hundred page book.

What is more is that, as concerns the problem of writing, at least in certain cases, the process is reversible. Taking a macro-proposition, it is possible to rediscover its underlying micro-propositions.

In the domain of computer programming an analogous distinction has been made between an expert's and a novice's representation of a program (which is a kind of text). Abelson (1984) shows that the novice's representation remains at the detailed level of "how a program functions". On the other hand, the expert's representation is at the level of "what a program does": this is an abstract level (a macro-structure level) which no longer contains the details of the process.

Thus we can conceive in a more precise way the fuzzy notion of "idea" used in the studies on writing. An idea may well be considered as having a relative autonomy; ideas can be viewed as macro-propositions with varying degrees of abstraction. In particular each idea can be expanded at a more specific level.

\section{Representing relations}

The second question concerns the possibility of concretely representing the semantic relations that the writer manipulates in order to organize ideas coherently and hierarchically.

I think that this would not be a correct objective. Specific semantic relations are studied currently in different scientific fields such as knowledge representation, argumentation, explanation. But the results seem still too incomplete and widely dispersed. And above all, even if possible, it is unlikely that providing the writers with representations of specific semantic relations that they would have to manipulate would be an aid to them .Too many possible semantic relations would be a source of complexity and extra constraints rather than of efficient aid.

I believe that aiding system, at least for a first generation, should keep to the only few semantic relations that are exhibited by the writers' observable behavior, both in their final texts (see Tazi \& Virbel, 1985a) and in the notes they produce (Hayes \& Flower op. cit.). Actually there is a small set of very general relations between ideas which would be worth taking into account; 
these are mainly the sequence relation, the hierarchical relation and the relation of set membership .

\section{Available computer aids for planning}

Is it possible to find tools that aid planning in this way? As far as I know, only plan editors are available on the market. But I do not believe that these editors are really aiding the planning process because they are based on a strictly hierarchical top-down model.

Here is an example of a software which - in order not to advertise - I name "Idea-lab" available on the "Raincoat" computer. On the first page of the Idealab-manual it says:

" the first program that offers the user the power of a personal computer to structure and organize his ideas".

According to the manual this software "enables the user to organize his loose ideas by forming hierarchies and arranging them by topics". The principle is compared with that of Russian dolls in order to highlight the power that the user can expect from Idea-lab!

Idea-lab is faithful to the top-down model to such an extent that the manual underlines that:

"it is not possible to shift a title more than one level to the And: right of the title that preceeds it".

"it is forbidden to shift a title to the left of the one that immediately follows it".!

And, of course, you cannot start in another way than in writing the general title (the very first prompt is "UNTITLED") 1 .

In fact, I personnally very much appreciate using Idea-lab on my Raincoat computer; but only for what it can help to do and that is editing plans that are already well designed. But it is claiming far too much to say that it aids the plan design process itself.

However, some researchers have moved towards this direction.

Nanard et Al. (1984) for example, have designed a computer-aided system the explicit aim of which is to set documents containing texts and illustrations. But the principles upon which their tool is founded seem suitable for ideas as well.

They use a box model. A document is a hierarchical tree made up of boxes. A box is either a terminal object or else a group of boxes. Nonetheless they explicitly choose to al!ow "a mixed strategy" which is "sometimes top-down and sometimes bottom-up". Thus they offer the users two ways of manipulating the boxes:

1 Two "devices" constrain you to start with the title: plan editors and ... conferences organizing committees! 
- on the one hand they can create a hierarchical tree of boxes, in the same way as in a classic plan editor,

- but on the other hand they can also create boxes that are independent the one from the other and store them, temporarily, in a "box stack".

In this stack the users may regroup the boxes in a bottom-up way. So they can move boxes, or groups of boxes in both directions: either from the tree to the stack, or from the stack to the tree.

Brown and Newman (1985) published some information about two tools they are experimenting on. The general principle that guides their design is "to reify the stages of thought in the authoring process by giving concrete form to the products of each stage".

With the first tool, their objective is explicitly helping the writers in their process of "formulation, clarification, and structuring of ideas". Here again the tool allows the users to create individualized notes on the screen. They can "shrink" these notes in order to concentrate on the relationships between groups of notes. To do this they can draw boxes that group sets of ideas and arrows that represent the relationships between them.

The second tool is designed for what the authors consider as a later phase, in which the writers expand their ideas in pieces of text. The machine provides the possibility of representing these pieces of text in the form of cards between which links can be established. The tool makes it possible to explicitly describe these links. The authors believe this to be an interesting possibility because "typing of links enables a separation between the semantic content of an individual idea and its relationship with other ideas".

One of the interesting possibilities of these tools may be that they could help the writers to distinguish more clearly in their whole activity between the process of manipulating ideas and the process of translating these ideas into a textual form.

It is however necessary to take into account the continuous interaction between all the sub-processes, which was brought to light by the research into writing.

It is true that ideas are generated faster than they can be coherently expressed; this is why writers have to resort to a kind of shorthand in their notes in order to reduce the constraints of linguistic problems.

But it is also true that only when writing out certain ideas do the writers discover exactly what they want to say and how this ties-in with other ideas. Often the expansion of one idea leads to the creation of new ideas and reorganizations of the plan .

It therefore seems necessary, I believe, that aids for the different subprocesses should not be presented by different tools. One single tool would allow the users both to distinguish the sub-processes and to move freely from one sub-process to another. 


\section{COMPUTER AIDED TRANSLATING}

I am focusing now on the translating process. This process allows an internal representation of an idea or of a set of ideas to be embodied in a well formed sentence or group of sentences.

The autonomy of the translating process has been confirmed experimentally. McCutchen (1986) carried out an experiment in which she asked children to write texts. She varied the grades and the degree of knowledge in the topic (which was football).

The results are that knowledge of the content plays an important role: the children produced a much more coherent text when dealing with a topic that they knew well. However, keeping the level of topic knowledge equal there were still important differences in the coherence of the texts, according to the grades.

The author concludes that "as children become more linguistically sophisticated, they acquire generalizable discourse and linguistic skills that they can use even when, and perhaps especially when, their topic knowledge is sparse".

Moreover it is now accepted that the internal representation of knowledge differs from its representation using language.

For one thing, the internal representation is more complete than its written or spoken expression. Writers rely, in fact, on the knowledge that the readers already have, in order to tell them only the minimum they need .

For another, the internal representation does not take the form of sentences. The units of internal representation are semantic propositions of the predicatearguments type, that are grouped into larger units that take into account their relations: these larger units are schemas, knowledge frames, scripts or semantic networks.

This structural aspect of knowledge is being widely studied at present both in Psychology and Artificial Intelligence. But what about the process aspect?

Although this process has indeed an autonomous existence, there are practically no psychological studies, as far as I know, that attempt to describe it directly.

On the other hand, a relatively important activity is, at present, being developed in Artificial Intelligence under the name of "text generation".

It concerns the generation of sentences to form short texts, usually of about one paragraph long. Knowledge structures, such as frames or schemas are used as inputs ior the generatiting system.

Some try, using natural language, to answer questions asked by data base users (McKeown, 1985). Others are attempting to describe events either in the style of an encyclopedia (Thompson, 1984) or else in journalistic style 
(Danlos, 1984, 1985). Another example of domain is the description of photographs of natural scenes (Conklin \& McDonald, 1982).

These studies do not try to model the translating process of human subjects and therefore their authors do not attempt to test their psychological validity. But the great interest of these researches is that they result in programs which do produce texts and that, to achieve this, the authors are obliged to clearly define, and find solutions to, all the problems that this translating process poses.

Without being able to go into detail here, let us say that they have all defined and solve two main categories of problem:

- firstly the problems of the necessary decisions about content. For a given semantic representation what information can - or must remain implicit, and what information must be explicit in the text? and in what order should this information be presented?

For example, to solve these problems in a scene description system, Conklin and McDonald (1982) carried out experiments, asking the subjects to rate the salience of the various items that made up the scenes represented in the photographs. In other experiments they asked the subjects to provide written descriptions of the same photographs.

They found that the degree of salience is "quite sensitive to changes in the size and centrality of objects in the scene". They also notice a strong correlation between the order of the objects according to a decreasing salience and the order in which the objects are mentioned in the written descriptions. The authors use the results to design "a program that generates descriptive paragraphs comparable to those produced by people".

- secondly the problems of linguistic decisions. Which words from the lexicon are to be selected? Which syntactic constructions are to be used? How is the text to be divided into sentences?

The text generator designers use in particular what they name "presentation strategies" that are domain-independent frames, each one corresponding to a specific linguistic objective such as: a "define $X "$ frame; a "describe $X$ " frame; a frame "analogy of $X$ to $Y$ "; etc.

A specially interesting perspective is given by Danlos (1985) in reaction against modular approaches that treat decisions about content and linguistic decisions separately.

Danlos shows that "decisions about lexical choice, determination of the order of the information, segmentation into sentences and choice of syntactic construction are all dependent on one another".

To account for this she introduces in her generator on the one hand what she calls a "lexicon-grammar" which includes both phrases specific to the domain and their associated syntactical properties, on the other hand a "discourse grammar" which integrates all the linguistic decisions. 
Such a discourse-grammar specifies the relation between meaning and form; it establishes a correspondance between one semantic relation (for example the causal relation that the author treats in detail) and the list of structures that allow it to be expressed. A discourse grammar "provides all the combinaisons that are both formally realisable and semantically appropriate".

The author states in conclusion that although the results are coherent texts, with a correct syntax and an pleasant style, the amount of work necessary to have such texts eventually produced is very large. Not only is the lexicon specific to the domain, but a discourse grammar is specific to a given semantic relation. To construct such a grammar demands a "deep linguistic study of the possibilities offered by the language to translate the semantic relation concerned". Danlos states that this line of research, although potentially very productive, is necessarily very lengthy.

However, from the point of view of possible aid for the human translating process this is the only way I can imagine. It is the way of an expert system.

Two cases could be considered: in one case the users would be able to indicate what they want to say by filling in slots in the schemas or frames specific to the domain. This would constitute the input to the text-generator which would then carry out the process of transforming these elements of information into a written text in correct language. If need be, for certain sentences for example, it could produce several possibilities from which the users could choose one.

In the other case the users would write their text and the expert-system would complete the revision process, proposing improvements (see Vaughan \& McDonald, 1986).

Certainly this is not going to be possible for many texts for a long time to come; and unfortunately I don't believe that we shall be able to use such systems for our scientific texts in the near future. But I do not think it unreasonable to imagine that such systems may well exist before too long for certain restricted (and well confined) domaines. A good example that is of particular interest to cognitive ergonomics is of course that of writing manuals for hardware and/or software.

\section{CONCLUSION}

Deliberately this paper has not included any part on text editors. Because the editing process is currently the only process that is really computer-aided the objective was to look for possibilities to aid more comprehensively the whole process of text production.

In such a perspective it is not only a matter of adding new aids but also of allowing the writers the complex intermixing of the sub-processes the researches on writing have highlighted. To make such an integrated tool and even for the only editor component the designers should be less narrowly confined to a restricted view of editing. The current view is still too much inspired by the task of the typist which consists in recopying a text already composed. 
In their review on the "Behavioural aspects of text editors", Embly and Nagy (1981) criticized the view of editing process as "a transformation from an existing string of symbols known as the source file (which in the case of initial text entry, may be null) to a new string of symbols, known as the target file". This has not changed a lot. To acheive this transformation the word editors offer a set of functions, which are practically always the same.

That which is different and what most of the studies try to improve, is the dialogue mode that is supplied to the users in order to put these functions into operation. The main criteria are usability and learnability. I do not deny the interest of this work: the recent innovation of the direct manipulation has been remarkable, and by the way it is a good example of cooperation between computer science and ergonomics.

However even if it is now far easier, it remains that the users have to continuously command what they want at a very low level. Research should be emphasized towards a greater computer involvment.

An exemplary resarch on this way is that of Tazi and Virbel (1985a) whose project is to produce an intelligent text editing and formatting system in which human expertise may be represented. Thus their project is aimed at allowing the writers to avoid specifying their intentions using commands to inform the system about the final representation of their text. To do this the authors undertook a preliminary study in order to analyse all the natural marks that writers spontaneously introduce when writing either on a typewriter or by hand. Their working hypothesis is that the text physical characteristics are non-discursive traces of meta-textual speech acts such as: introduce, entitle; enumerate, underline, etc.

The originality of this research is that, although it is focused on the editing sub process, it takes into account its relation to the other sub-processes in text composition.

The fact that the researches which focus on the man-machine dialogue are useful and successfull should not lead to neglect the necessary researches towards a more fundamental and integrated concept of assistance. It was the goal of this paper to suggest that theses researches should not be centered primarily on the tool (or the product) characteristics but above all on the analysis and modelling of the cognitive activities of the (potentially aided) writers.

\section{ACKNOWLEDGEMENTS}

I am grateful to Pierre Falzon, Jean François Richard and Willemien Visser who criticized first drafts of this paper and gave me very useful advices...specially about the (product) plan.

I want to thank Marie Pierre Laborne who helped to last editing and formatting sub-processes. 


\section{REFERENCES}

ABELSON, B. (1984) - When Novices Surpass Experts: The Difficulty of a Task May Increase With Expertise. Journal of Experimental Psychology: Learning, Memory and Cognition, Vol. 10, № 3, 483-495.

BISSERET, A. (1983) - Psychology for Man Computer Cooperation in Knowledge Processing. In R. E. A. Masson (Ed.): Information Processing 83. Amsterdam, North Holland, 1983.

BROWN J. S. \& NEWMAN, S. E. (1985) - Issues in Cognitive and Social Ergonomics: From Our House to Bauhaus. Human-Computer Interaction, 1985, vol. 1, pp. 359-391.

CARROLL, J. M. \& ROSSON, M. B. (1985) - Usability Specifications as a Tool in Iterative Development. In H. R. Hartson (Ed.), Advances in HumanComputer Interaction, Vol. 1. Ablex Publishing Corporation, 1985.

COLLINS, A. \& GENTNER, D. (1980) - A Framework for a Cognitive Theory of Writing. In L. W. Gregg \& E. R. Steinberg (Eds.), Cognitive Processes in Writing. Hillsdale, LEA, 1980.

CONKLIN, E. J. \& MCDONALD, D.D. (1982) - Salience: the Key to the Selection Problem in Natural Language Generation. In Proceedings Twentieth Annual Meeting of Association for Computational Linguistics;Toronto, 16-18 June 1982, pp. 129-135.

DANLOS, L. (1984) - Conceptual and Linguistic Decisions in Generation. In Proceedings Tenth International Conference on Computational Linguistics, Stanford CA.; 2-6 June 1984, pp. 501-504.

DANLOS, L (1985) - Génération automatique de textes en langues naturelles. Paris, Masson, 1985.

EMBLEY, D. W. \& NAGY, G. (1981) - Behavioral Aspects of Text Editors. ACM Computing Surveys, Vol. 13, № 1, 1981, pp. 33-70.

GOULD, J. D. (1980) - Experiments on Composing Letters: Some Facts, Some Myths, and Some Observations. In L. W. Gregg \& E. R. Steinberg (Eds.), Cognitive Processes in Writing, Hillsdale, LEA, 1980.

HAYES J. R. \& FLOWER L. S. (1980) - Identifying the Organization of Writing Processes. In L. W. Gregg \& E. R. Steinberg (Eds.), Cognitive Processes in Writing. Hillsdale, LEA, 1980.

HAYES-ROTH, B. \& HAYES-ROTH, F. (1979) - A Cognitive Model of Planning. Cognitive Science, 3, 275-310.

HOC, J. M. (1987) - Psychologie cognitive de la planification. Presses Universitaires de Grenoble, 1987. 
KINTSCH, W. \& VAN DIJK, T. A. (1978) - Toward a Model of Text

Comprehension and Production. Psychological Review, 1978, vol. 85, N 5, pp. 363-394.

MALHOTRA, A., THOMAS, J. C., CARROLL, J. M. \& MILLER, L. A. (1980) Cognitive Processes in Design. International Journal of Man-Machine Studies, 1980, 12, 119-140.

MCCUTCHEN, D. (1986) - Domain Knowledge and Linguistic knowledge in the Development of Writing Ability. Journal of Memory and Language, $25,431-444$.

MCKEOWN, K. R. (1985) - Discourse Strategies for Generating NaturalLanguage Text. Artificial Intelligence, 27, 1985, 1-41.

MORAN, T. P. (1981) - The Command Language Grammar: A Representation for the User Interface of Interactive Computer Systems. International Journal of Man-Machine Studies, 1981, 15, 3-50.

NANARD, M., NANARD, J. \& FALGUEIRETTES, J. (1984) - Top down or Bottom up Approach for Document Structuration. Centre de Recherche en Informatique de Montpellier, research report $N^{\circ} 15$.

REISNER, P. (1984) - Formal Grammar as a Tool for Analysing Ease of Use: Some Fundamental Concepts. In J. C. Thomas \& M. L. Schneider (Eds.), Human Factors in Computing Systems. Ablex Publishing corporation, 1984.

SACERDOTI, E. D. (1977) - A Structure for Plans and Behavior. Elsevier, 1977.

TAZI, S. \& VIRBEL, J. (1985a) - Formal Representation of Textual Structures for an Intelligent Text-Editing System. In V. Dahl \& P. Saint-Dizier (Eds.), Natural Language Understanding and Logic Programming, pp. 191-205. Elsevier, North Holland, 1985.

TAZI, S. \& VIRBEL, J. (1985b) - The Limits of the Hierarchical Model for Textual Representation. Research Report LSI, Université Paul Sabatier, Toulouse, 1984.

THOMAS, J. C. \& CARROLL, J. M. - Human Factors in Communication. IBM Systems Journal, Vol. 20, $N^{\circ} 2,1981$.

THOMPSON, C. W. (1984) - Object-Oriented Text Generation. In Proceedings First Conference on Artificial Intelligence Application. Denver, Colorado, 5-7 dec. 1984, pp. 524-529. IEEE Computer Society Press, 1984.

VAN DIJK, T. A. (1977) - Semantic Macro-Structures and Knowledge Frames in Discourse Comprehension. in Mi. A. Just \& P. A. Cârpenter (Eds.), Cognitive Processes in Comprehension. Erlbaum, 1977. 
VAUGHAN, M. M. \& McDONALD, D. D. (1986) - A Model of Revision in Natural Language Generation. In Proceedings Twenty fourth Annual Meeting of the Association for Computational Linguistics. New York, 10-13 July 1986.

VIRBEL, J. (1985) - Représentation et utilisation de connaissances textuelles. In Actes du colloque Cognitiva 85, pp. 879-883. Paris, Cesta,1985.

VISSER, W. (1987) - Abandon d'un plan hiérarchique dans une activité de conception. Actes du colloque Cognitiva 87; Paris, Cesta, 1987. 
